\title{
Using Market Indicators to Eliminate Local Trends for Financial Time Series Cross-Correlation Analysis
}

\author{
Zohreh Alamatian ${ }^{1}$, Majid Vafaei Jahan ${ }^{1, *}$, Amin Milani Fard ${ }^{2}$ \\ ${ }^{1}$ Islamic Azad University, Mashhad, Iran \\ ${ }^{2}$ New York Institute of Technology, Vancouver, Canada
}

\begin{abstract}
Multifractal detrended cross-correlation analysis (MFDCCA) is largely used to analyze non-stationary financial time series. Existing methods for such analysis utilize the time series itself as the detrending function with a polynomial. We propose a technique for a more accurate removal of local trends, called indicator-based MFDCCA (IMFDCCA), which leverages market technical indicators to better determine correlations between financial time series. We evaluated our method on pair trading in the Foreign Exchange Market (Forex) and our results show that the proposed IMFDCCA compared to the MFDCCA reduces the RMSE for the Hurst exponent estimation by $30 \%$.
\end{abstract}

Keywords: time series, multifractal, cross-correlation analysis, local trend, indicators

\section{Introduction}

Transactions in financial markets generate huge amount of data in the form of time series that reflect price changes. Cross-correlation analysis of two financial time series helps to understand the impact of changes amongst interconnected components of the market [1]. Correlated time series can be used for predicting and decision-making in financial markets. Currency pairs, such as EUR/USD or USD/JPY, are key variables in financial markets as their fluctuations influence banks, traders, policy makers, and economic institutions [2], and thus determining such currency pairs relation can help in market prediction.

Most financial time series are complex and non-stationary, i.e., they do not fluctuate around a fixed average and have varying mean and variance. Statistical techniques, such as vector autoregressive or correlation, which are used for linear correlation analysis of price fluctuations, are not appropriate for the analysis of nonlinear and non-stationary behavior of financial time series and thus fractal analysis techniques are used [3]. The dynamic characteristic of a complex system can be specified by fractal property based on the scaling exponent that is a criterion for analysis of their self-similarity and helps in predicting the system behavior. Monofractal time series have a fixed scale, meaning that a fractal pattern is repeated without considering the scale factor, and so there is no difference in their shape at different zoom levels. Changes in most time series, however, cannot be determined by one scaling exponent. To capture the dynamism of such systems a multifractal is used that is determined by multiple scaling exponents at different sections of the series also called the singularity spectrum. Examples of multifractal systems are weather changes, and economic series. Multifractal analysis enables us to investigate the long-term behavior of time series with respect to each other and determine whether the multifractal relation will continue at longer time scales which in turn helps in prediction and decision making.

Short-term behaviors of financial markets are affected by external factors, such as important events, while long-term behaviors are influenced by internal market factors. Peng et al. [4] presented the Detrended Fluctuation Analysis (DFA) technique to discover fractal structure, however, it could not be used for multifractal analysis. Kantelhardt et al. [5] proposed the Multifractal DFA (MFDFA) which became popular in long-term autocorrelation

${ }^{*}$ Corresponding author: vafaeijahan@mshdiau.ac.ir

This article is ( 2021 by author(s) as listed above. The article is licensed under a Creative Commons Attribution (CC BY 4.0) International license (https://creativecommons.org/licenses/by/4.0/legalcode), except where otherwise indicated with respect to particular material included in the article. The article should be attributed to the author(s) identified above. 
detection. Later Podobnik et al. [6] extended DFA for detrended cross-correlation analysis (DCCA) to determine cross-correlation between two non-stationary time series. Zhou combined DFA and DCCA and proposed MFDCCA [7] that since then is largely used for multifractal analysis of financial time series such as [8].

Detrending financial time series helps to determine their correct volatility and fluctuations by eliminating the effects that might be related to sudden events. Existing techniques have used the intended time series to create the function for removing local trends. In this work we incorporate the effect of influential external factors using technical indicators of the market, and use the indicator function as a fitness function for removing local trends. Technical indicators of markets are functions that use open, close, maximum, and minimum price, and trading volume, to help in forecasting the future trend of the market. Our hypothesis is that using technical indicators that reflect the market movement trend can assist in a more accurate elimination of local trends in time series. In this preliminary work we only focus on multifractal analysis methods and do not compare our solution with sequence learning methods. We propose the Indicator-based Multifractal Detrended CrossCorrelation Analysis (IMFDCCA) technique that leverages technical market indicators, such as ATR, in the detrending step of MFDCCA [7].

\section{The Proposed Approach}

We explain the five steps of our IMFDCCA, for which the first two steps are the same as DCCA [6], our novelty for using polynomials of technical indicators to eliminate local trends is presented in step 3, and the rest follows MFDCCA [7].

Step 1: Let $x(i)$ and $y(i)$ be two non-stationary time series, where $i=1,2, \ldots, N$, and $N$ is the maximum number of samples or length of the series. We determine the profile (cumulative series) of them as $X(i)=\sum_{t=1}^{i} x(t)-\bar{x}$, and $Y(i)=\sum_{t=1}^{i} y(t)-\bar{y}$, where $\bar{x}=\frac{1}{N} \sum_{t=1}^{N} x(t)$, and $\bar{y}=\frac{1}{N} \sum_{t=1}^{N} y(t)$.

Step 2: $X(i)$ and $Y(i)$ are divided into $N_{s}=\operatorname{int}(N / s)$ nonoverlapping segments of equal length $s$. Since the length $N$ of most time series is often not a multiple of the time scale $s$, for not disregarding the remaining part, the same dividing procedure is repeated this time starting from the end of the series. Thus, $2 N_{s}$ segments are obtained in total.

Step 3: For each segment $v$ of length $s$, we calculate the local trend by a least-square fit of $X(i)$ and $Y(i)$, using a polynomial function of order $n$, which represents the trend in that segment. This step is to determine the local trend in each segment to be removed later. We then calculate the covariance of the residuals in each section:

$$
F_{v}^{2}=\frac{1}{s} \sum_{i=1}^{s}\left|X_{v}((v-1) s+i)-\bar{x}_{v}(i)\right| \cdot\left|Y_{v}((v-1) s+i)-\bar{y}_{v}(i)\right|,
$$

where $X_{v}$ and $Y_{v}$ are the fitting polynomials in segment $v=1, \ldots, 2 N_{s}$.

In this work, however, instead of using polynomial functions for fitting the data, we propose leveraging equations of technical market indicators for detrending. The movement of such indicators can reflect the direction of financial markets. For example, the Average True Range (ATR) indicator defines the true ranges of the trade in a simple form:

$$
T R_{t}=\max \left[\left(\text { high }_{t}-\operatorname{low}_{t}\right), \operatorname{abs}\left(\text { high }_{t}-\text { close }_{t-1}\right), \operatorname{abs}\left(\text { low }_{t}-\text { close }_{t-1}\right)\right],
$$

which measures market volatility by decomposing the entire range of an asset price for that period. The true range indicator is taken as the maximum of the following: current high less the current low; the absolute value of the current high less the previous close; and the absolute value of the current low less the previous close. ATR is a moving average, typically using 14 days, of the true ranges. The indicator formula at time $t$ is $A T R_{t}=$ $\frac{A T R_{t-1} *(n-1)+T R_{t}}{n}$ and then ATR is calculated as $A T R=\frac{1}{n} \sum_{i=1}^{n} T R_{i}$. 
In our proposed method, for the data in each segment $v$ at time $t$, the value of ATR indicator is calculated and the interpolation polynomial function is determined according to this indicator. The ATR time series is then fitted to a degree-2 polynomial to be used for local detrending in the covariance function $F_{v}^{2}$.

Step 4: We average over segments to get the $q$-th order fluctuation function:

$$
\begin{aligned}
& F_{x y}(q, s)=\left[\frac{1}{2 N_{s}} \sum_{v=1}^{2 N_{s}} F_{V}(S)^{q / 2}\right]^{1 / q} ; q \neq 0 \\
& F_{x y}(0, s)=\exp \left[\frac{1}{4 N_{s}} \sum_{v=1}^{2 N_{s}} \ln F_{V}(s)\right] ; q=0
\end{aligned}
$$

For $q=2$, the standard DCCA procedure is retrieved. In general, we are interested to know how the fluctuation function depends on the time scale.

Step 5: We repeat the above steps for different time scales and determine the scaling of the fluctuation function by analyzing $\log -\log$ plots of $F_{x y}(q, s)$ versus $s$ for each $q$. For two cross-correlated time series $x$ and $y$, there is a power-law relation between the fluctuation function $F_{x y}(q, s)$ and the scale $s$ as $F_{x y}(q, s) \sim s^{h_{x y}(q)}$.

For very large values of $s, F_{x y}(q, s)$ becomes statistically unreliable as the number of segments $N_{s}$ for the averaging procedure in step 4 becomes very small. If series $x$ and $y$ have exponential long-term correlation, the covariance function increases exponentially for large values of s. When $x$ and $y$ are equal, this turns into MFDFA. Note that the long-term correlation between time series means that current changes in one series depends on the past changes in the other one.

The Hurst Exponent. The Hurst exponent is used to explain autocorrelation or selfsimilarity at different time scales. Different methods have been proposed to estimate this exponent and we also proposed one. By taking logarithm from both sides of the above equation, we obtain the Hurst exponent for each scale:

$$
h_{x y}(q)=\frac{\log \left(F_{x y}(q, s)\right)}{\log (s)}
$$

For $q=2$ this analysis turns into the DCCA, and $0<h_{x y}(2)<0.5$ indicates negatively correlated, $h_{x y}(2)=0.5$ implies not correlated, and $0.5<h_{x y}(2)<1$ means stronger correlation. Cao et al. [9] studied that the dependency of $h_{x y}(q)$ to $q$ can be used to explain the behavior between two time series. If $h_{x y}(q)$ changes with different values of $q$, it indicates that the relation between the time series is multifractal and monofractal otherwise. The scaling exponent $h_{x y}(q)$ represents the degree of the cross-correlation between the time series. $h_{x y}(q)=0.5$ denotes the absence of cross-correlation. $h_{x y}(q)>0.5$ indicates persistent long-range cross-correlations, i.e., a large value in one variable is likely to be followed by a large value in another variable. $h_{x y}(q)<0.5$ implies anti-persistent cross-correlations, i.e., a large value in one variable is likely to be followed by a small value in another variable.

\section{Evaluation}

We implement our method in MQL4 on the MetaTrader 4 trading platform and make the code and our dataset available for download ${ }^{1}$. We assess the efficacy of our IMFDCCA against MFDCCA on determining long-term and short-term relations of currency pairs EUR/USD and USD/JPY. The reason for choosing MFDCCA as the baseline is that using technical indicators (consist of 5 time series) has limited us to not being able to consider common evaluation methods that consider a specific Hurst Exponent. Also related recent works such as [1] did not change steps of MFDCCA and thus were not comparable.

\footnotetext{
$1_{\text {https }} / / /$ figshare.com/s/282ffd3b47acff02fb9b
} 
Dataset and Data Collection. We extracted our dataset from the history center of MetaTrader for FXTM broker (www.forextime.com) containing 2000 records of 8 years data from October 10, 2011 to September 18, 2019 for currency pairs EUR/USD and USD/JPY. In our experiments we used the logarithmic returns $r_{\Delta t}=\log (p(t+\Delta t))-\log (p(t))$, where $\Delta t$ denotes the returns' time-lag the daily close price of currency pairs.

Comparison. We compare the Hurts exponent calculated by our method against two most common Hurst exponent estimation techniques R/S [10] and GHE [11, 12]. To do so, we calculate the Hurst exponent in each time series with these methods and then use the following equation from [13] to determine the overall Hurst exponent for time series:

$$
h_{x y}=\frac{h_{x x}+h_{y y}}{2}
$$

This is the average of the generalized Hurst exponent of time series $x$ and $y$ at scale $q$, and and it corresponds to the Hurst exponent $H$ for $q=2$.

Rescaled Range Method. Hurst [10] proposed this method and is still commonly used for fractal time series analysis. According to this method, for a time series $x$ of length $n$, the rate of $R(n) / S(n)$ is defined as: $\left[\max \left(x^{c u m}(t, n)\right)-\min \left(x^{\text {cum }}(t, n)\right)\right] / \sqrt{\frac{1}{n-1} \sum_{t=1}^{n}(x(t)-\bar{x})^{2}}$, where $x^{c u m}(t, n)=\sum_{i=1}^{t} x(i)-\bar{x}(n)$ and $\bar{x}(n)=\frac{1}{n} \sum_{t=1}^{n} x(t)$, i.e., $R(n)$ is the range of cumulative deviate series $x^{\text {cum }}(t, n)$, and $S(n)$ is the standard deviation of $x$. Finally, the Hurst exponent is defined as: $H=\frac{\log \left(\frac{R}{S}\right)}{\log n}$.

Generalized Hurst Exponent Method. GHE method is the extended version of the classic Hurst exponent. It is the main method to calculate the exponent for long-term relation analysis of time series [11,12] and is used to determine multifractal behavior. Let: $K_{q}(\tau)=$ $\frac{\left\langle|X(t+\tau)-X(t)|^{q}\right\rangle}{\left\langle|X(t)|^{q}\right\rangle}$, where $1<\tau<\tau_{\max }$ determines the number of periods in time series, and $<$.$\rangle is the average within the time window. Then GHE is K_{q}(\tau) \propto \tau^{q H(q)}$, and the Hurst exponent is defined as: $H=\frac{\log K_{q}(\tau)}{\log \tau}$.

\subsection{Results}

We compare the Hurst exponent in equation 3.1 with the ones obtained by our method and the baseline and report the root mean square error as $R M S E=\sqrt{\left.\frac{\sum_{q=-10}^{10}\left(H_{x y}(q)\right.}{21}-h_{x y}(q)\right)^{2}}$. Our results show that for the short-term relation of $H(q)$ versus $q$ for EURUSD and USDJPY, the RMSE for MFDCCA(Linear fit) is 0.173309 and for IMFDCCA (ATR fit) is 0.125028. Also for the long-term relation of $H(q)$ versus $q$ for EURUSD and USDJPY the RMSE for MFDCCA(Linear fit) is 0.144925 and for IMFDCCA (ATR fit) is 0.078993 . This indicates that our method yields a smaller error.

Fig. 1 presents the calculated exponents using IMFDCCA and MFDCCA in shortterm and long-term for EUR/USD and USD/JPY, and shows changes of exponent $h(q)$ with respect to $q$. This indicates that IMFDCCA predicts the relation of two time series to be multifractal as $h(q)$ changes with $q$, whereas MFDCAA [7] predicts the long-term relation to be monofractal. We further analyze the fractal property with respect to each other by evaluating the data volume used in cross-correlation analysis. Figure 2 shows long-term and short-term relationships with different data lengths and scales. In the longterm, the monofractal relationship between the two time series is observed in the MFDCCA method, while in the IMFDCCA the result is multifractal. As explained in the introduction section, such fractal relations can have a major impact in trading and market prediction. The multifractal relation that is obtained by the proposed method has a smaller error and indicates that the movement of a currency pair can affect the other pair in the long term. 


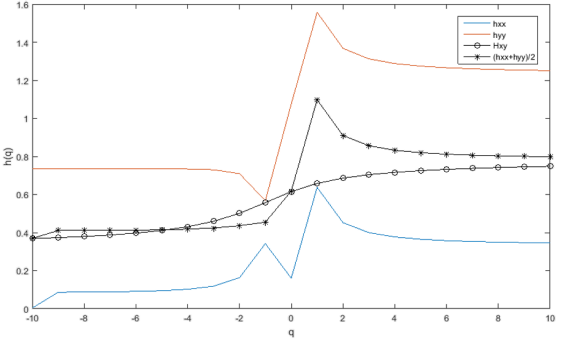

(a) Short-term with IMFDCCA

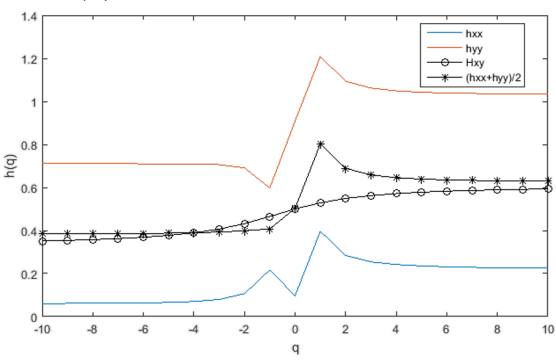

(c) Long-term with IMFDCCA

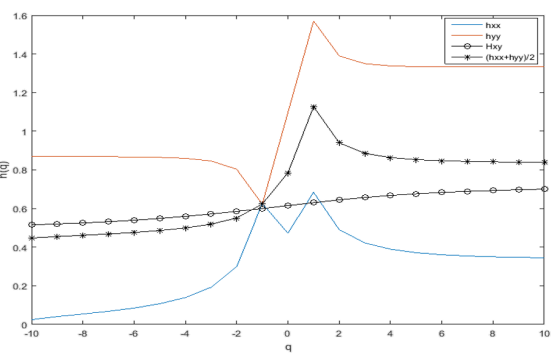

(b) Short-term with MFDCCA

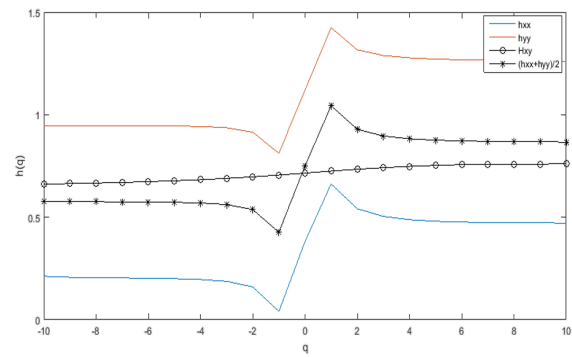

(d) Long-term with MFDCCA

Figure 1. Hurst exponent in short-term and long-term for EUR/USD and USD/JPY

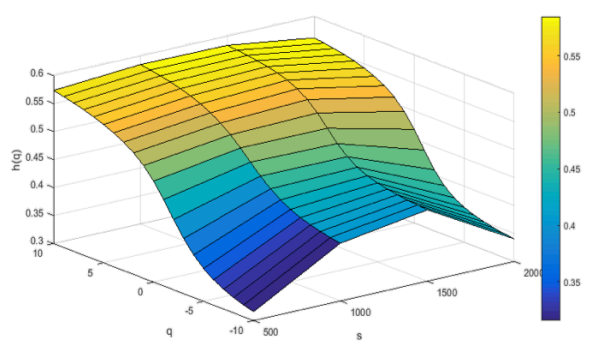

(a) IMFDCCA (ATR fit)

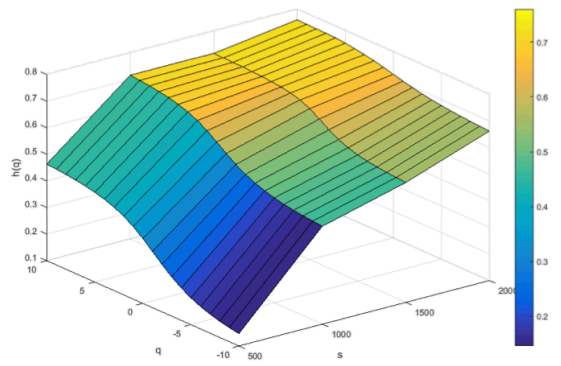

(b) MFDCCA (Linear fit)

Figure 2. $h_{x y}(q, s)$ plotted with different $q$ and $s$ values showing multifractal and monofractal relation of two time series with different lengths

Table 1. Comparison against the Rescaled Range and the Generalized Hurst Exponent methods.

\begin{tabular}{l|l|l|l|l} 
Length of time series $(n)$ & IMFDCCA $(q=2)$ & MFDCCA $(q=2)$ & R/S & GHE $(q=1)$ \\
\hline \hline 500 & 0.50114 & 0.39581 & 0.50628 & 0.36154 \\
\hline 1000 & 0.52109 & 0.63508 & 0.52797 & 0.53343 \\
\hline 1500 & 0.52490 & 0.69504 & 0.53735 & 0.54685 \\
\hline 2000 & 0.49258 & 0.73403 & 0.65136 & 0.59003 \\
\hline \hline RMSE w.r.t R/S & $\mathbf{0 . 0 7 9 7 4}$ & 0.11519 & & \\
\hline RMSE w.r.t GHE & $\mathbf{0 . 0 8 6 0 5}$ & 0.11640 & &
\end{tabular}

Table 1 shows the comparison of calculated Hurst exponent using MFDCCA and IMFDCCA methods. Note that we set $q=1$ for the GHE method as suggested in [14]. Results indicate that our proposed method has reduced the root-mean-squared error compared to the base method by $30 \%$ and $26 \%$ using $\mathrm{R} / \mathrm{S}$ and GHE respectively. 
Table 2. Multifractal degree (MF) for EURUSD versus other currency pairs.

\begin{tabular}{l|r|r|r|r|r|r|r} 
& USDCHF & GBPUSD & USDCAD & AUDUSD & EURJPY & XAUEUR & XAUUSD \\
\hline \hline MF Degree & 0.247780 & $\mathbf{0 . 3 3 0 9 6 3}$ & 0.179011 & 0.239952 & 0.231616 & 0.180248 & $\mathbf{0 . 2 8 1 2 9 9}$
\end{tabular}

Lastly we investigate the strength of multifractal relations between EUR/USD and other currency pairs by calculating the multifractal degree that implies the range of the Hurst exponent [15]. Table 2 shows EUR/USD has stronger multifractal relations with GBP/USD and XAU/USD compared to others.

\section{Conclusions and Future Work}

We proposed a method to remove local trends by leveraging market indicators for multifractal cross-correlation analysis, which results in a more accurate detrending in different parts of time series. We plan to investigate other influential series functions for detrending and also consider using sequence learning techniques to deal with the temporal dynamics.

\section{References}

[1] W. Shi, P. Shang, J. Wang, and A. Lin. "Multiscale multifractal detrended cross-correlation analysis of financial time series". In: Physica A: Statistical Mechanics and its Applications 403 (2014), pp. 35-44.

[2] R. J. Elliott, J. Van Der Hoek, and W. P. Malcolm. "Pairs trading". In: Quantitative Finance 5.3 (2005), pp. 271-276.

[3] J. W. Kantelhardt. "Fractal and multifractal time series". In: arXiv:0804.0747 (2008).

[4] C.-K. Peng, S. V. Buldyrev, S. Havlin, M. Simons, H. E. Stanley, and A. L. Goldberger. "Mosaic organization of DNA nucleotides". In: Physical review e 49.2 (1994), p. 1685.

[5] J. W. Kantelhardt, S. A. Zschiegner, E. Koscielny-Bunde, S. Havlin, A. Bunde, and H. E. Stanley. "Multifractal detrended fluctuation analysis of nonstationary time series". In: Physica A: Statistical Mechanics and its Applications 316.1-4 (2002), pp. 87-114.

[6] B. Podobnik and H. E. Stanley. "Detrended cross-correlation analysis: a new method for analyzing two nonstationary time series". In: Physical review letters 100.8 (2008), p. 084102.

[7] W.-X. Zhou. "Multifractal detrended cross-correlation analysis for two nonstationary signals". In: Physical Review E 77.6 (2008), p. 066211.

[8] H.-Y. Wang and T.-T. Wang. "Multifractal analysis of the Chinese stock, bond and fund markets". In: Physica A: Statistical Mechanics and its Applications 512 (2018), pp. 280-292.

[9] G. Cao, Y. Han, W. Cui, and Y. Guo. "Multifractal detrended cross-correlations between the CSI 300 index futures and the spot markets based on high-frequency data". In: Physica A: Statistical Mechanics and its Applications 414 (2014), pp. 308-320.

[10] H. E. Hurst. "Long-term storage capacity of reservoirs". In: Trans. Amer. Soc. Civil Eng. 116 (1951), pp. 770-799.

[11] A.-L. Barabási and T. Vicsek. "Multifractality of self-affine fractals". In: Physical Review A 44.4 (1991), p. 2730.

[12] B. B. Mandelbrot. Fractals and scaling in finance: Discontinuity, concentration, risk. Selecta volume E. Springer Science \& Business Media, 2013.

[13] X. Zhang, L. Yang, and Y. Zhu. "Analysis of multifractal characterization of Bitcoin market based on multifractal detrended fluctuation analysis". In: Physica A: Statistical Mechanics and its Applications 523 (2019), pp. 973-983.

[14] M Fernandez-Martinez, M. Sánchez-Granero, and J. T. Segovia. "Measuring the self-similarity exponent in Lévy stable processes of financial time series". In: Physica A: Statistical Mechanics and its Applications 392.21 (2013), pp. 5330-5345.

[15] Y. Yuan, X.-t. Zhuang, and Z.-y. Liu. "Price-volume multifractal analysis and its application in Chinese stock markets". In: Physica A: Statistical Mechanics and its Applications 391.12 (2012), pp. 3484-3495. 